\title{
La peau de Chagrin de Honoré de Balzac. Valores ideológico- culturales prototípicos del culturema literario. Estudio multilingüístico.
}

\author{
Lucía Luque Nadal \\ Universidad de Córdoba \\ fe1lunal@uco.es \\ https://dx.doi.org/10.12795/futhark.2011.i06.07
}

\begin{abstract}
This paper describes how both idioms and culturemes can be used to describe ideological and prototypical schemes of a language and a culture. The use of idioms and culturemes also involves the assumption of pragmatic and semantic elements embedded in them. It is therefore necessary to explain how these elements behave in the creation of discourse. We analyze the ideological and cultural meanings of the expression "the wild ass's skin" in several languages.

Resumen: En este trabajo se expone la manera en que tanto fraseologismos como culturemas pueden utilizarse para designar esquemas ideológicos prototípicos de una lengua y una cultura. La utilización de un culturema o un fraseologismo lleva consigo también la asunción de una serie de elementos pragmáticos y semánticos insertos en estos. Por ello es necesario explicar cómo funcionan dichos elementos en la creación del discurso. En concreto, se estudian los valores ideológicos y culturales que posee una misma expresión, "la piel de zapa", en varios idiomas.
\end{abstract}

Key words: culturology, culturemes, idioms, pragmatics, semantics

Palabras clave: culturología, culturemas, fraseologismos, pragmática, semántica

\section{Introducción}

Este trabajo se enmarca dentro de las investigaciones llevabas a cabo por el Grupo de Investigación de Lingüística Tipológica y Experimental 
(GILTE) de la Universidad de Granada relacionadas con la inclusión de los denominados 'culturemas'1 en los Diccionarios Interlingüísticos e Interculturales².

Los estudios de los investigadores del grupo GILTE han demostrado que la presencia de culturemas en distintas lenguas es muy elevada. Los culturemas se encuentran especialmente en el lenguaje de los media: textos periodísticos y argumentativos, debates televisivos, películas, pero también en textos literarios, chistes, anuncios, etc ${ }^{3}$.

Por diversas razones, los culturemas no han sido aún estudiados de una manera sistemática ni tampoco se incluyen generalmente en los diccionarios de uso. Una de las razones que contribuyen a esta situación es la falta de acuerdo que existe sobre qué tipo de información 'cultural' se ha de inventariar en los diccionarios culturales y de qué forma se ha de hacer. Una propuesta pionera que ofrece respuestas al respecto se encuentra en Luque Durán ${ }^{4}$. Esta obra junto con la obra monográfica de Luque $\mathrm{Nadal}^{5}$ constituyen las bases teóricas sobre las que trabaja el grupo GILTE en la actualidad.

Además, los culturemas suponen a menudo un serio escollo tanto para estudiantes como traductores de lenguas extranjeras. EI estudio de los culturemas es incluso una materia importante para el buen conocimiento de la lengua materna, dado que el carácter culto o

1. Encontramos multitud de definiciones de culturema según la escuela que lo defina. Aunque el término tiene su origen en el ámbito de la traducción, aquí desarrollamos el término culturema desde la perspectiva de los estudios lingüístico-culturales que entienden culturema como "cualquier elemento simbólico cultural específico, simple o complejo, que corresponde a un objeto, idea, actividad o hecho, que sea suficientemente conocido entre los miembros de una sociedad para que pueda utilizarse como medio comunicativo y expresivo en la interacción comunicativa de los miembros de esa cultura".

${ }^{2}$. Los Diccionarios Interlingüísticos e Interculturales son una nueva apuesta lexicográfica iniciada por el grupo GILTE que pretende rellenar el hueco que existe actualmente en la lexicografía actual conformado por la ausencia de diccionarios en los que se dé cuenta de los elementos culturales que componen una determinada lengua y por extensión una determinada cultura.

${ }^{3}$. Los investigadores del grupo GILTE parten de la premisa de que lo 'cultural' está interrelacionado con lo lingüístico y que la cultura aflora en hechos lingüísticos.

${ }^{4}$. Juan de Dios Luque Durán: "La codificación de la información lingüístico-cultural en los diccionarios (inter)culturales". En Luque Durán y Pamies Bertrán (eds.): Interculturalidad y Lenguaje I. El significado como corolario cultural. Granada 2007, pp. 329-374.

5. Lucía Luque Nadal: Fundamentos teóricos de los diccionarios lingüístico-culturales. Relaciones entre fraseología y culturología. Granada 2010. 
literario de muchos culturemas hace que estos constituyan una barrera para muchos hablantes nativos cuya educación ha sido deficiente en este aspecto 6 .

Por todo ello, los Diccionarios Interlingüísticos e Interculturales elaborados por miembros del GILTE, pretenden:

-Explicar el origen y funcionamiento de los culturemas, especialmente aquellos que han dado lugar a un 'esquema ideológico prototípico'.

- Seleccionar y estudiar los culturemas más frecuentemente usados de cada lengua.

-Facilitar la comparación de culturemas de distintas lenguas, estableciendo no solo equivalencias, coincidencias, diferencias parciales, etc., sino también los 'falsos amigos culturológicos'.

- Integrar los culturemas junto a los fraseologismos en un marco lingüístico culturológico amplio que pueda exponerse en diccionarios, métodos de enseñanza de idiomas, etc.

\section{Los culturemas: culturemas materiales y culturemas proposicionales.}

De las diferentes definiciones de 'culturema' se deriva la existencia de al menos dos tipos generales de culturemas: los culturemas materiales y los culturemas proposicionales ${ }^{7}$.

Los culturemas materiales derivan de la definición tradicional del término propuesta por Vermeer ${ }^{8}$ quien define culturema como "Un

6. Piénsese por ejemplo en la utilización en la prensa de culturemas clásicos como Saturno devora a sus hijos o históricos como la campana de Huesca. Si el lector español desconoce los referentes no podrá comprender el significado del texto ni tampoco el valor pragmático crucial que estos culturemas tienen en la estructuración de la argumentación.

7. Para un extenso estudio y clasificación de los culturemas véase Lucía Luque Nadal: "Los culturemas: ¿unidades lingüísticas, ideológicas o culturales? Language Design 11, 2009, pp. 93-120.

8. Hans J. Vermeer: "Translation theory and linguistics". En P. Roinila, R. Orfanos \& S. Tirkkonen-Condit, eds. Häkökohtia käänämisen tutkimuksesta. Joensuu 1983, pp. 1-10; aquí p. 8. 
fenómeno social de una cultura A que es considerado relevante por los miembros de esta cultura y que, cuando se compara con un fenómeno social correspondiente en la cultura $B$, se encuentra que es específico de la cultura A".

De otra parte, siguiendo la definición de culturema ofrecida por Luque Durán" ${ }^{9}$, quien indica que los culturemas son "unidades semióticas que contienen ideas de carácter cultural con las cuales se adorna un texto y también alrededor de las cuales es posible construir discursos que entretejen culturemas con elementos argumentativos", se deriva que algunos culturemas poseen una mayor complejidad semántica y pragmática. Bajo esta definición de culturema se englobarían los culturemas proposicionales.

Culturemas materiales ${ }^{10}$ son aquellos que representan objetos. Así en español: tapa, tortilla, paella, gazpacho, siesta, fandango, flamenco; en ruso: borsch, samovar, isba, kulak, dacha, koljós; en japonés: sake, kimono, samurai, geisha; en árabe: kasbah, fez, cuscus, henna, faluca, fellah, arrack, kibla, cadi, dhow, douar, etc.

En general, los culturemas materiales son estáticos, se refieren a objetos propios de una cultura, bien sean comidas, vestimenta, vivienda, diversiones, objetos religiosos, etc. Los culturemas materiales remiten, por tanto, a otro universo de experiencias, sabores, imágenes.

Los culturemas proposicionales tienen un carácter dinámico o cualitativo y evocan hechos y personajes que al ser aludidos nos remiten a situaciones prototípicas. Estas situaciones suelen caracterizarse por la interrelación de personajes en condiciones tales que vemos en ellos modelos ejemplares para interpretar nuestras propias acciones y situaciones. Así, este tipo de culturemas se nos presenta como unos "esquemas ideológicos prototípicos" dentro de los cuales existe una red de actantes prototípicos, relaciones prototípicas, etc. El valor cognitivo y

\footnotetext{
9. Juan de Dios Luque Durán: "El Diccionario Intercultural e Interlingüístico y su aplicación a la traducción de culturemas". En Ortega Arjonilla, E. y Marçalo, M.J. (eds.), Lingüística et Traduçao na Sociedade do Conhecemento. Evora 2009, pp. 177-188.

${ }^{10}$. Como es lógico, un elemento material puede convertirse en un elemento ideológico y de ahí ser generalizado y aplicado a otras situaciones. Así por ejemplo, el 'burka', en principio, es un elemento de la vestimenta femenina. Por diversas razones ha pasado a ser considerado en Europa un elemento de 'represión', a veces de una manera genérica: "el burka de las monjas católicas", "el burka de tierra", etc.
} 
argumentativo que poseen los "esquemas ideológicos prototípicos" radica en el hecho de que son trasladables a hechos y situaciones actuales que mediante la traspolación a un "esquema ideológico prototípico" quedan revisualizados y reevaluados con una nueva óptica. Un ejemplo de este proceso de reevaluación parte del uso del "esquema ideológico prototípico" que se encierra en la expresión francesa Paris vaut bien une messe ${ }^{11}$. Esta expresión ha permeado a través del francés a otras lenguas europeas. A partir de la expresión francesa se crean expresiones en alemán: Paris ist eine Messe wert; en inglés: Paris is worth a mass; en español: París bien vale una misa; en ruso: Париж стоum мессы; en italiano: Parigi val bene una messa; en portugués: Paris vale uma missa, etc.

Luque $\mathrm{Nadal}^{12}$ expone que este culturema aparece frecuentemente en la prensa y realiza el siguiente análisis del mismo:

NOTICIA: "Soraya Sáenz de Santamaría ironiza con la visita de Zapatero a Washington en un desayuno religioso. 'Hay que ver dónde vamos a ver a un laico. Obama bien vale una misa ${ }^{13}$.

COMENTARIO: Este texto funciona siempre que el lector conozca la frase original 'París bien vale una misa' que subyace al mismo, y posteriormente realice la siguiente operación mental:

1. El presidente del gobierno de España, Zapatero, viaja a Washington a un desayuno religioso.

2. El presidente Zapatero es un laico que ha dejado siempre clara su postura contraria a la religión.

11. Esta expresión se le atribuye al rey Enrique IV de Borbón (1553-1610) quien, a fin de conseguir el trono de Francia, renegó de sus creencias protestantes convirtiéndose al catolicismo.

12. Lucía Luque Nadal: "Traducir culturemas o cómo enfrentarse a la traducción de términos y expresiones específico-culturales". En López-Campos Bodineau, Balbuena Torezano, C. y Álvarez Jurado, M. (eds), Traducción y modernidad. Textos científicos, jurídicos, económicos y audiovisuales. Córdoba 2010, pp. 275-284; aquí pp. 279-281.

13. http://www.elplural.com/politica/detail.php?id=42288 
3. Si el presidente Zapatero asiste a una ceremonia religiosa es porque considera su relación con el presidente Obama de mayor interés que sus convicciones.

El hecho de utilizar un "esquema ideológico prototípico" para reevaluar una acción presente permite al político 0 al periodista aplicar y superponer un elemento adicional a una acción que ha ocurrido. En este caso, el rasgo que se superpone es el de [OPORTUNISMO]. El "esquema ideológico prototípico" de París bien vale una misa se aplica a aquellas acciones que el argumentador tacha de 'oportunistas' porque en ellas el interés coyuntural prima sobre las creencias o convicciones personales ${ }^{14}$.

\subsection{La importancia del tratamiento lexicográfico de los culturemas.}

Como se ha indicado anteriormente, hoy en día, en los diccionarios disponibles tanto para los traductores como para los estudiantes de lenguas extranjeras, no se presta adecuada atención al registro ni a la explicación de los denominados 'culturemas' 15 .

Es necesario que tanto el traductor como el estudiante de lenguas extranjeras conozcan a grandes rasgos el origen, significado y aplicaciones prácticas de un culturema ya que, si no es así, no podrán comprender actos lingüísticos diversos relacionados o derivados del mismo, como por ejemplo variaciones jocosas, chistes, desviaciones intencionadas, etc., que se hagan a partir de dichos culturemas ${ }^{16}$.

14. Para utilizar con propiedad estas expresiones complejas desde el punto de vista pragmático y semántico es necesario que se inventaríen y que se explique y clarifique en cada una de ellas cómo, cuándo y para qué se han de utilizar.

15. Uno de los ejemplos más parecidos, aunque limitado, lo encontramos en obras como la de Michael Ferber, Dictionary of Literary Symbols. Cambridge 1999. No obstante, este diccionario solo se centra en explicar aquellos símbolos recurrentes en la literatura pero no profundiza en el estudio de otros símbolos mucho más actuales que aparecen en la prensa, en textos argumentativos, en el discurso político, etc.

16. Aunque un diccionario de símbolos o expresiones específicas de un ámbito concreto pudiera ofrecer una significación de una determinada expresión, esta información seguiría estando incompleta ya que no ofrecería información alguna sobre aquellos aspectos pragmáticos relativos a la producción y al contexto de producción de dicha expresión. 
Como se ha podido comprobar a través de nuestras investigaciones, muchos culturemas aparecen la mayoría de las veces alterados, es decir, se les cambia uno o varios de sus elementos con un fin lúdico o argumentativo determinado. Este reemplazo es fácilmente superable por el hablante/lector nativo que, en general, puede reponer sin dificultad los términos elididos o modificados. Por esto, es necesario conocer no solo el valor idiomático de un culturema dado sino también la 'historia que subyace a este' para poder comprender por completo su uso en determinados textos en los que aparece alterado en alguno de sus componentes.

Además, cada culturema debe aparecer en los diccionarios acompañado de información semántica y pragmática relativa a en qué situaciones se usa, qué intencionalidad se persigue, etc. En resumen, al analizar cada culturema en nuestros Diccionarios Interlingüísticos e Interculturales se sigue un esquema que se compone de varios niveles de análisis de los cuales destacamos aquí la comparación de los culturemas en diferentes lenguas.

\section{Comparación de culturemas en diferentes lenguas}

Los "esquemas ideológico- prototípicos" se muestran en las lenguas en forma de fraseologismos o culturemas que representan la sabiduría de los conocimientos acumulados de una sociedad y de una cultura. Toda cultura está basada en conocimientos clásicos, literarios, populares, religiosos, etc. En cada país existen culturemas propios y culturemas compartidos, es decir, existen una serie de culturemas que traspasan fronteras y se encuentran en muchas lenguas. Por esta razón, además de clasificar los culturemas según su procedencia habrá que analizar su universalidad o particularidad. Para la comparación translingüística de los culturemas es necesario disponer y analizar diferentes ejemplos de su uso en las distintas lenguas.

Para ilustrar este punto tomaremos como ejemplo de estudio un culturema francés proveniente de la literatura: peau de chagrin. Esta expresión tiene su origen en el título de la obra homónima de Honoré de Balzac escrita en el año $1831^{17}$. Para comprender el significado de esta

17 La versión consultada es Honoré de Balzac: La peau de chagrin. Paris 1839. 
expresión y el valor ideológico prototípico subyacente a esta habría que resumir brevemente el argumento de la obra.

La obra versa sobre un hombre llamado Raphaël de Valentin quien, en un momento de desesperación tras haberse arruinado por el juego se plantea firmemente la posibilidad de suicidarse arrojándose al Sena. Con este firme propósito en mente y al deambular por las calles de París se cruza en su camino una pintoresca tienda donde un vendedor le ofrece una extraña pieza de piel que tiene el poder de conceder todo lo que se desee. No obstante, a la par que el trozo de piel de zapa concedía todo aquello que su dueño quería, la piel encogía y robaba energía vital a su dueño, según estaba escrito en la piel de zapa en lengua árabe ${ }^{18}$. El protagonista hace caso omiso de las advertencias sobre el poder maligno que la piel de zapa tenía sobre la duración de la vida de aquel que le pedía deseos. Al final de la obra el protagonista acaba muriendo prematuramente por su desmesurada necesidad de pedir deseos a la piel de zapa.

Esta obra de Honoré de Balzac tuvo un gran éxito en su época. Este éxito se demuestra actualmente en el hecho de que una obra teatral escrita en 1831 todavía hoy sea un referente actual el cual incluso haya dado origen a expresiones fraseológicas en francés ${ }^{19}$.

Un estudio cultural y lingüístico del culturema 'peau de chagrin' implica, como se ha mencionado anteriormente, comprobar si la expresión francesa 'peau de chagrin' ha traspasado fronteras, al igual que lo ha hecho la novela de Honoré de Balzac ${ }^{20}$.

Tomando algunas lenguas europeas comprobamos la existencia de las siguientes expresiones: Esp. piel de $z^{2} a^{21}$; Ing. wild ass's skin; Al. ein Chagrinleder ; Rus. Шагреневая кожа.

18. En el original francés se lee: Si tu me possèdes, tu posséderas tout. Mais ta vie m'appartiendra. Dieu l'a voulu ainsi. Désire, et tes désirs seront accomplis. Mais règle tes souhaits sur ta vie. Elle est là. A chaque vouloir je décroîtrai comme tes jours. Me veux-tu? Prends. Dieu t'exaucera. Soit!

19. De ahí que la expresión peau de chagrin en francés signifique 'algo que mengua, que disminuye de tamaño' en modismos como rétrécir comme une peau de chagrin, lit. 'menguar como una piel de zapa'.

20. Esta obra de Honoré de Balzac influenció al compositor alemán Giselher Wolfgang Klebe en su ópera titulada Die tödlichen Wünsche (1958-1959).

21. No existe ninguna referencia a la expresión piel de zapa en el Diccionario fraseológico documentado del español actual de Manuel Seco, Olimpia Andrés y Gabino Ramos, 
Una vez comprobada la existencia de estas expresiones, se ha de proceder a una comparación interlingüística sistemática de los valores que dichas expresiones tienen en sus respectivas lenguas para comprobar si el valor del culturema francés 'peau de chagrin' se mantiene inalterable en las demás lenguas o bien, si por el contrario, a partir de su significado básico han derivado algún otro significado secundario.

Para ello ilustraremos cada ejemplo con un texto real tomado de la prensa y de textos argumentativos. Dichos textos serán puestos en contexto y serán comentados a fin de hallar las similitudes con el culturema origen 'peau de chagrin'.

Comenzamos con el análisis de un texto francés en el cual se ve el uso actual de la expresión 'peau de chagrin'22 :

\section{TEXTO 1 [FRANCÉS]}

L'influence peau de chagrin de la France à Bruxelles ${ }^{23}$

Juste avant les vacances, j'avais souligné l'impressionnante perte d'influence des Français au sein de la Commission européenne : ils sont réduits à la portion congrue au niveau des plus hauts postes administratifs (directeurs généraux et directeurs généraux adjoints), notamment au profit des Allemands, des Britanniques ou encore des... Irlandais. Cette perte d'influence se confirme lorsqu'on examine la composition des cabinets des 27 commissaires européens, des postes éminemment politiques.

CONTEXTO: En este artículo el autor se queja de la reducción de representantes franceses que existen actualmente en la Comisión Europea, que si bien han sido limitados a los más altos cargos, al

Madrid 2004 ni tampoco en el Diccionario de fraseología española de Jesús Cantera Ortiz de Urbina y Pedro Gomis Blanco, Madrid 2007. No obstante ambos diccionarios dan cuenta de la expresión trabajo de zapa.

22. En el diccionario Le Bouquet des expressions imagées de Claude Duneton y Sylvie Claval, Paris, 1990, p. 741, se define la expresión se réduire (se rétrécir) comme une peau de chagrin como "diminuer peu à peu jusqu'à presque rien. Par allusion au roman de Balzac".

23. http://bruxelles.blogs.liberation.fr/coulisses/2009/09/linfluence-peau-de-chagrin-de-lafrance-\%C3\%A0-bruxelles-suite.html 
descender el número de representantes, otros países como Alemania, Gran Bretaña o incluso Irlanda están aumentando peligrosamente su número de representantes y acercándose al número de representantes franceses.

COMENTARIO: El título del artículo ya contiene en sí mismo el mensaje que posteriormente desarrolla el periodista: la comparación entre la idea de la piel de zapa y la situación actual de Francia en la Comisión Europea de Bruselas. Según el periodista, el número de representantes ha ido disminuyendo año tras año por cuestiones políticas relacionadas con el conocimiento nativo del inglés. Poco a poco países como Irlanda han ido aumentando su cupo en Bruselas gracias a su lengua nativa. Sin embargo, esto parece ser una paradoja, pues, en opinión del periodista, cuando más anglosajona se vuelve Europa menos es querida por estos.

En español encontramos el siguiente texto en el que se utiliza la expresión 'la piel de zapa'24 :

\section{TEXTO 2 [ESPAÑOL]}

Los valencianos arrancarán 2008 con subidas de luz, gas, peajes ${ }^{25}$.

El sueldo de los valencianos corre el riesgo de sufrir el destino del dueño de una piel de zapa. Honoré de Balzac escribió en 1831 La piel de zapa. La novela cuenta la historia de Rafael, propietario de un extraño trozo de piel que mengua conforme cumple sus deseos. Al ritmo que se encoge la piel, mengua la vida del protagonista, hasta que muere.

Aunque es lógico pensar que el destino final del consumidor no sea tan dramático como el de Rafael, es cierto que las nóminas de 2008 pueden acabar escribiéndose en una peligrosa piel de zapa.

24. Si buscamos en el DRAE el término 'zapa' no encontramos alusión alguna al valor derivado de la novela de Balzac. Zapa es definido como:

Zapa (Del lat. sepia, lija).

1. f. Piel áspera de algunos selacios.

2. f. Piel labrada de modo que la flor forme grano como el de la lija.

3. f. Labor que en obras de metal imita los granos de la lija.

25. http://www.lasprovincias.es/valencia/20071230/tema-dia/valencianos-arrancaran-2008subidas-20071230.html 
CONTEXTO: El periodista informa en esta noticia acerca de las constantes subidas de impuestos que los habitantes de la Comunidad Valenciana sufrirían irremediablemente en el año 2008. Tal fueron las subidas en todos los servicios que el periodista supone que los sueldos de los valencianos menguarán como la piel de zapa de la novela de Balzac.

COMENTARIO: La utilización de la imagen de la piel de zapa en este artículo periodístico se fundamenta en la idea de que una piel de zapa es algo que constantemente va menguando de tamaño. En el caso de artículo son los sueldos de los habitantes de la Comunidad Valenciana lo que va menguando constantemente por las subidas en todos los servicios y aspectos del día a día.

En inglés el culturema aparece bajo la forma the wild ass's skin ${ }^{26}$ :

\section{TEXTO 3 [INGLÉS]}

\section{Is Stupid Making Us Google? James Bowman²7}

Immersing myself in a book or a lengthy article used to be easy. My mind would get caught up in the narrative or the turns of the argument, and l'd spend hours strolling through long stretches of prose. That's rarely the case anymore. Now my concentration often starts to drift after two or three pages. I get fidgety, lose the thread, begin looking for something else to do. I feel as if I'm always dragging my wayward brain back to the text. The deep reading that used to come naturally has become a struggle." Sound familiar? Describing, in The Atlantic Monthly, his own struggles to keep his attention span from contracting like the wild ass's skin in Balzac's novel, Nicholas Carr cites a British study of research habits among visitors to two serious scholarly websites which suggests a more general problem: that "users are not reading online in the traditional sense; indeed there are signs that new forms of 'reading' are emerging as users 'power browse'

26. La traducción literal del término francés chagrin en inglés es shagreen, término que se utiliza para referirse a una piel de tacto áspero que se obtenía del caballo o del onagro. De este último se ha tomado la traducción de la expresión en inglés, así como del título de la novela de Balzac. Un onagro (en inglés onager) es un asno salvaje.

27. http://www.thenewatlantis.com/publications/is-stupid-making-us-google 
horizontally through titles, contents pages and abstracts going for quick wins. It almost seems that they go online to avoid reading in the traditional sense."

CONTEXTO: El articulista lanza en este artículo la idea de que los nuevos métodos de búsqueda de información, entre ellos el más destacado el buscador Google, esté influyendo negativamente en nuestra manera de leer y comprender los textos. Según James Bowman, Google nos está volviendo quizá cada vez más ineptos para leer y comprender un texto puesto que gracias a su sistema de búsquedas y presentación de la información estamos acostumbrándonos a leer horizontalmente, buscando la información pertinente en vez de profundizar en todas las palabras del texto.

COMENTARIO: La utilización de la imagen de la piel de zapa en este artículo periodístico es muy acertada ya que la piel de zapa se correspondería aquí con nuestra atención a la hora de leer un texto. Conforme más utilizamos el buscador Google, nuestra atención va menguando hasta llegar al punto de no poder leer un texto completo palabra por palabra pues queremos obtener el máximo de información en el mínimo de tiempo.

El alemán ha tomado la expresión bajo la forma sintética ein Chagrinleder :

\section{TEXTO 4 [ALEMÁN]}

VDI nachrichten: Glauben Sie, dass Deutschland reformierbar ist?28

Leysen: Ich möchte Deutschland mit dem Mann aus Honoré de Balzacs Roman vergleichen, der ein Chagrinleder besitzt und sich davon allerlei Wünsche erfüllen lassen kann. Doch bei jedem Wunsch schrumpft das Leder. Irgendwann stellt man fest, dass es kein Chagrinleder mehr gibt. So ergeht es derzeit auch Deutschland: das Chagrinleder ist aufgebraucht. Deutschland wird jetzt mit der harten Realität konfrontiert. In wenigen Jahren wird Deutschland nur noch ein Prozent der Weltbevölkerung ausmachen und an Einfluss verlieren. Andererseits ${ }^{28}$.http://www.vdi-nachrichten.com/vdi-nachrichten/aktuelle_ausgabe/
article.asp?cat=2\&id=17499\&source=archiv 
glaube ich, dass Deutschland genügend Realitätssinn besitzt, um noch rechtzeitig umzusteuern.

CONTEXTO: En esta entrevista a un industrial alemán el entrevistador pregunta al mismo si Alemania está a tiempo de realizar reformas económicas que eviten pérdidas graves al país.

COMENTARIO: El industrial entrevistado responde a la pregunta sobre una posible reforma económica en Alemania y sobre el potencial que dicho país tendría a realizarla mediante una comparación curiosa: el industrial ve a Alemania como el dueño de la piel de zapa de la novela de Honoré de Balzac. Tanto Rafael, el dueño de la piel de zapa, como Alemania, han podido hasta ahora cumplir con facilidad todos sus deseos. Pero al igual que en la novela de Balzac, la piel de zapa, es decir, el potencial económico alemán tiene un límite que va menguando conforme se van cumpliendo los deseos y aspiraciones económicas del país. Según el industrial, Alemania está en el límite de caer en una gran crisis económica y perder la influencia económica que hasta ahora ha tenido, pero todavía está a tiempo de recuperarse.

La utilización en esta entrevista de la imagen de la piel de zapa para referirse a los recursos económicos presumiblemente inagotables de Alemania crea una metáfora bastante acertada, pero en el caso de Alemania con un final más prometedor y no tan negativo, pues en palabras del industrial, el país todavía puede salvarse si se comienzan a realizar medidas de contención y recuperación económica.

Finalmente, en ruso también existe la misma expresión bajo la forma шагреневая кожа :

\section{TEXTO 5 [RUSO]}

\section{Шагреневая кожа экономики 29}

Невероятно много всего хочется российским властям. И саммита АТЭС-2012, и Олимпиады-2014, и Чемпионата мира по футболу-

29. http://www.gazeta.ru/column/mikhailov/3372106.shtml 
2018 или 2022, и строительства избыточных мощностей по газопроводам, и новых нефтепроводов (явно убыточных), и консервации сложившейся государственно-коррупционной структуры, и спасения собственности олигархов, и модернизации экономики, и увеличения социальных выплат населению, и собственных перевыборов...

Экономика пока выполняет все желания властей, но условия для ведения бизнеса сокращаются, как шагреневая кожа.

CONTEXTO: En este artículo de un experto ruso del Centro de Estudios Económicos y Políticos, este tacha la actual política económica de Rusia como la 'economía de la piel de zapa' como reza el título en el original (Шагреневая кожа экономики). Según el experto, el gobierno ruso quiere conseguir muchos logros para el país (entre los que destacan la Cumbre APEC 2012, los Juegos Olímpicos de 2014, o la Copa Mundial FIFA 2018 o 2022 todo ello con el incremento de la capacidad en los nuevos gasoductos y oleoductos, junto con una serie de reformas sociales para promover la reelección del gobierno actual. Todas estas promesas se quieren intentar cumplir, sin importar que haya o no crisis.

COMENTARIO: El experto en economía tilda acertadamente los planes del gobierno de ser una mera 'economía de la piel de zapa' ya que actualmente todavía la economía rusa puede realizar sin problemas los múltiples deseos de las autoridades pero como ocurre con la piel de zapa, las condiciones económicas para hacer tantos negocios va menguando poco a poco hasta quedar agotada y corre el riesgo de quedar exhausta al igual que la piel de zapa de la novela de Balzac, por no haber sabido controlar los deseos y los gastos.

\section{Conclusiones}

El estudio sistemático de miles de fraseologismos y cientos culturemas en diferentes idiomas desarrollado por el grupo GILTE de la Universidad de Granada permite contrastar los culturemas de diferentes idiomas 
estableciendo su igualdad, proximidad, equivalencia más o menos matizada, etc., además de los llamados 'falsos amigos culturológicos' ${ }^{30}$.

El culturema 'piel de zapa', como se ha indicado, se encuentra en diferentes lenguas europeas. No obstante, en el caso propuesto para el alemán y el ruso, el valor ideológico-cultural de la expresión 'la piel de zapa' está más estrechamente ligado a la expresión original francesa ya que en ellas entran en juego dos ideas: de una parte la idea de 'algo que mengua irremediablemente' pero también la causa de que ese algo mengüe, es decir, la avaricia de gobernantes que quieren bien obtener favores políticos o bien obtener logros económicos sin pensar que la fuente que les provee pueda agotarse en un futuro no muy lejano. En el caso del ejemplo español o del ejemplo inglés, la idea básica con la que se juega es con la idea de algo que va menguando hasta casi desaparecer (en el ejemplo español se habla de los sueldos de los ciudadanos, en el ejemplo inglés de la capacidad de los lectores de procesar mediante la lectura un texto completo).

En resumen, el análisis de los usos de la expresión 'peau de chagrin' en español, inglés, francés, alemán y ruso nos muestra que básicamente este culturema tiene en estas lenguas el mismo valor semántico y pragmático.

No siempre ocurre lo mismo y en el estudio contrastivo de fraseologismos y culturemas a menudo constatamos diferencias notables del mismo culturema en diferentes lenguas. El estudio translingüístico de los fraseologismos y los culturemas nos ofrece multitud de ejemplos de diferencias semánticas y pragmáticas más 0 menos señaladas de los valores de estos y por tanto de la existencia de 'falsos amigos'31. Así por ejemplo, el francés y el español conceptualizan de manera diferentes personajes bíblicos como Herodes o Job. Un

30. Aunque existen obras monográficas dedicadas al estudio de los falsos amigos en la fraseología, como por ejemplo la obra de María Rodríguez Rodríguez (coord.), Léxico, fraseología y falsos amigos, Madrid 2003, estos son trabajos que todavía no ofrecen una perspectiva completa a este respecto.

${ }^{31}$. Actualmente, la única forma que hay de detectar estos falsos amigos es mediante el estudio comparado de dos diccionarios de expresiones específicas (v.g. diccionario de expresiones bíblicas, de expresiones literarias). No existe todavía, por tanto, una obra lexicográfica que exponga las expresiones culturales en varias lenguas. 
ejemplo de falta de equivalencia entre fraseologismos españoles y franceses lo encontramos por ejemplo en las expresiones francesas vieux comme Hérode (Mateo 2, 1 y sq) y être pauvre comme Job (Job $1)^{32}$. En español Job es parangón de la paciencia y Herodes de la maldad. Así, las expresiones españolas agotarle alguien la paciencia al santo Job, tener más paciencia que el santo Job o más malo que Herodes.

Finalmente también puede ocurrir que en una lengua existan o sean más comunes una serie de culturemas que en otra lengua son casi desconocidos. Así por ejemplo, si comparamos el francés con el español comprobamos que en francés existen culturemas bíblicos desconocidos o muy poco usados en español ${ }^{33}$. Así por ejemplo: des jérémiades (Jérémie); un capharnaüm (Mateo 4, 12-14); le démon de midi (Salmo 91, 6); à chaque jour suffit sa peine (Mateo 6, 34); crier sur les toits (Mateo 10,27); tout travail mérite salaire (Luc 10, 7).

Por todo ello concluimos que el estudio cultural de los elementos lingüísticos supone una ardua tarea, como ya indicaba Sabban ${ }^{34}$, ya que no solo nos basta con el estudio de los culturemas de una determinada lengua sino que además hemos de comprobar cuántos de estos culturemas han traspasado fronteras y se han 'internacionalizado' frente a aquellos que son 'culturales específicos'. Además, sobre aquellos culturemas que existen en diversas lenguas se ha de realizar un estudio translingüístico como el llevado a cabo en este trabajo sobre la expresión 'peau de chagrin' para comprobar si todos ellos tienen el mismo significado o si bien cada cultura ha derivado significados pragmáticos secundarios.

32. Para un estudio en detalle de las diferencias entre las expresiones bíblicas en inglés y en español véase Lucía Luque Nadal: "Las referencias bíblicas en inglés y en español. Estudio culturológico- contrastivo". Revista Odisea, número 11, 2010.

${ }^{33}$. Esta idea fundamenta la opinión que las diferentes lecturas de la misma obra, en este caso la Sagrada Biblia, dan origen a diferentes expresiones. Estas expresiones por tanto varían según la cultura y/o la lengua de la que hablemos.

34. Annette Sabban: "Culture-boundness and problems of cross-cultural phraseology". En Burger, H., D. Dobrovol'skij, P. Kühn y N. R. Norrick (eds.): Phraseology. An International Handbook of Contemporary Research. Berlin 2007, pp. 590-605. 
